\title{
Concentration anomaly of heat capacity in PbTe based solid solutions
}

\author{
E.I. Rogacheva ${ }^{1}$, N.A. Sinelnik, I.M. Krivulkin

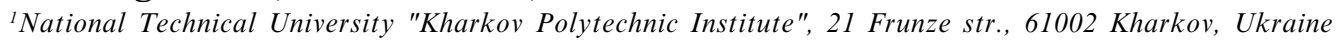

\begin{abstract}
The temperature dependences of the heat capacity in the $\mathrm{Pb}_{1-\mathrm{x}} \mathrm{Mn}_{\mathrm{x}} \mathrm{Te}$ and $\mathrm{Pb}_{1-\mathrm{x}} \mathrm{Ge}_{\mathrm{x}} \mathrm{Te}_{\mathrm{K}}$ $(x=0-0.04)$ solid solutions based on PbTe were obtained in the temperature range of $100-670 \mathrm{~K}$. Pronounced peaks were observed in the isotherms of the heat capacity in the vicinity of $x \approx 0.01-0.015$. The presence of the peaks is explained on the basis of the idea about the existence of concentration phase transitions of percolation type, which take place in any solid solution and are related to transformation of impurity discontinuum into impurity continuum.
\end{abstract}

Keywords: lead telluride, solid solution, heat capacity, temperature dependence, heat capacity isotherm, concentration phase transition, percolation.

Paper received 04.09.02; accepted for publication 17.12.02.

\section{Introduction}

In recent years, there has been considerable interest in studying the performance of semiconductor devices based on heavily doped semiconductors having highly degenerate carrier concentrations. This attracts attention to detailed studies of different properties of heavily doped semiconductors and the mechanism of the transition from slight to heavy doping. By now, it is the electron properties of heavily doped semiconductors that have been mostly investigated, whereas a number of works devoted to the lattice properties is insignificant. Under doping with isovalent impurities, no additional carriers are introduced into a semiconductor. However, atoms of the dopant disturb the crystal potential field thus affecting the lattice properties (phonon thermal conductivity, coefficient of linear expansion, heat capacity, microhardness) as well as the charge carrier mobility.

IV-VI semiconductor compounds and solid solutions on their basis are traditional materials for a wide variety of infrared optoelectronic devices. One of the basic methods of controlling properties of these materials is doping them with impurities.

It has been reported before [2-8] on the existence of anomalies in the concentration dependences of mechani- cal, thermal, galvanomagnetic and thermoelectric properties of $\mathrm{Pb}_{1-\mathrm{x}} \mathrm{Mn}_{\mathrm{x}} \mathrm{Te}$ and $\mathrm{Pb}_{1-\mathrm{x}} \mathrm{Ge}_{\mathrm{x}} \mathrm{Te}$ solid solutions in the range of concentrations $x=0.005-0.015$. The nonmonotonic character of the concentration dependences of properties has been interpreted in terms of our assumption $[9,10]$ about the existence of concentration-phase transitions of percolation type corresponding to the transition from an impurity discontinuum to an impurity continuum in any solid solution, regardless of the microscopic nature of interactions between particles. According to this assumption there should exist a critical concentration $x_{c}$ (percolation threshold), at which an uninterrupted chain of interactions between impurity atoms penetrating the whole crystal (infinite cluster) is formed.

According to the contemporary views [11-14] there is an apparent analogy between percolation phenomena and II-type phase transitions. Heat capacity is a universal physical property showing anomalous behavior in the vicinity of any II-type phase transition.

The aim of the present work is to prove the existence of such concentration phase transitions in $\mathrm{Pb}_{1-\mathrm{x}} \mathrm{Mn}_{\mathrm{x}} \mathrm{Te}$ and $\mathrm{Pb}_{1-\mathrm{x}} \mathrm{Ge}_{\mathrm{x}} \mathrm{Te}$ solid solutions by measuring heat capacity. 


\section{E.I. Rogacheva et al.: Concentration anomaly of heat capacity ...}

\section{Experimental}

The samples were prepared from high-purity elements (at least 99.999 at.\% of the main component) in evacuated down to $10^{-3} \mathrm{~Pa}$ graphitized quartz ampoules at $1200 \mathrm{~K}$ during 5-6 hours under vibrational mixing of the melt. Then the samples were subjected to homogenizing annealing at $T=820 \mathrm{~K}$ and subsequent cooling down with the switched-off furnace. A chemical analysis showed that the compositions of the samples corresponded to their nominal values within 0.03 at.\%. The results of microstructural analysis and X-ray diffractometry confirmed the existence of solid solutions in the entire range of concentrations investigated in this work.

The measurements of the heat capacity $C$ were performed on the samples with different compositions $(x=0-0.04)$ prepared by hot pressing the polycrystalline bulks and following annealing them at $820 \mathrm{~K}$ during 200 hours. All the samples were of p-type conductivity. The temperature dependences of the heat capacity in the range of 100-750 K were obtained for all samples. The heat capacity was measured using an adiabatic calorimeter in the regime of dynamic heating. A copper sample was used as a reference sample. For each sample at least three dependences $C(T)$ were obtained. The results were averaged. Since measurements of $C$ were carried out above the Debye temperature (for PbTe $Q_{D}=120 \mathrm{~K}[15,16]$ ), values of $C$ remained practically constant from $200-250 \mathrm{~K}$ and above. This increased the precision of measurements. As a result, values of $C$ for each sample were determined with accuracy not worse than $1.5 \%$. In Fig. 1, as an example, the temperature dependences of $C$ for the $\mathrm{Pb}_{1-\mathrm{x}} \mathrm{Mn}_{\mathrm{x}}$ Te samples of various compositions are given.
The points in the curves are the result of averaging values of 3-4 measurements.

The obtained values of $C$ for $\operatorname{PbTe}(C=165 \pm 3) \mathrm{J} / \mathrm{kg} \mathrm{K}$ are in good agreement with values reported in other works [17-20]. When calculating the lattice heat capacity, the contribution of the electronic heat capacity can be neglected already at $T>2-3 \mathrm{~K}$, since at $T=1 \mathrm{~K}$, the relative magnitude of the latter does not exceed 2-5\% [20]. That is why the obtained values of $C$ represent in fact lattice heat capacity.

\section{Results and discussions}

On the basis of the temperature dependences of the heat capacity, isotherms of $C$ were plotted for the $\mathrm{Pb}_{1-\mathrm{x}} \mathrm{Ge}_{\mathrm{x}} \mathrm{Te}$ and $\mathrm{Pb}_{1-\mathrm{x}} \mathrm{Mn}_{\mathrm{x}}$ Te systems (Fig. 2). In the isotherms the pronounced peaks of $C$ were observed in the vicinity of $x \approx 0.015$ and $x \approx 0.012$ for the $\mathrm{Pb}_{1-\mathrm{x}} \mathrm{Ge}_{\mathrm{x}} \mathrm{Te}$ and $\mathrm{Pb}_{1-\mathrm{x}} \mathrm{Mn}_{\mathrm{x}}$ Te systems, respectively.

The existence of the concentration anomaly of the heat capacity proves the assumption about some relation between observed concentration anomalies of different properties (microhardness, charge carrier mobility, Hall coefficient, etc.) [2-8] and critical phenomena, accompanying phase transitions of percolation nature.

According to contemporary views [11-14] percolation transitions have properties of II-type phase transitions. The formation of an infinite cluster in problems of percolation theory is similar to a II-type phase transition. Both Il-type phase transitions and percolation transitions have "universality regions" (near the Curie point and percolation thresholds, respectively), universal critical indices determining character of change of such physical char-

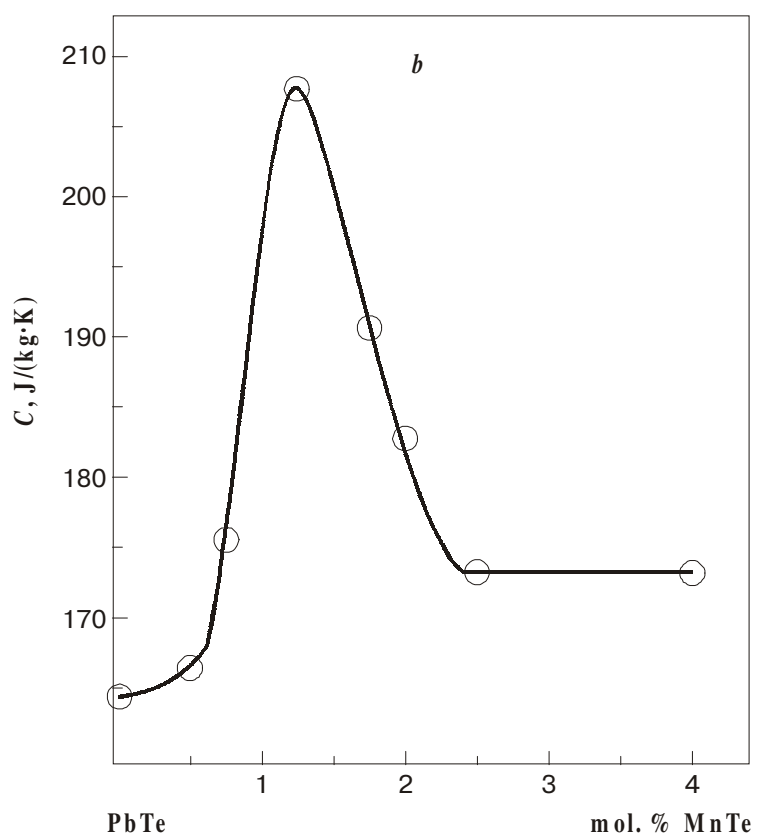

Fig. 1. The temperature dependences $(a)$ and the isotherm $(b)$ of heat capacity in the $\mathrm{Pb}_{1-\mathrm{x}} \mathrm{Mn}_{\mathrm{x}} \mathrm{Te}$ solid solutions: $1-\mathrm{PbTe} ; 2-$ 0.75 mol.\% MnTe; 3 - 1.25 mol.\% MnTe; 4 - 1.75 mol.\% MnTe; 5 - 2 mol.\% MnTe; 6 - 2.5 mol.\% MnTe; 7 - 4 mol.\% MnTe. 


\section{E.I. Rogacheva et al.: Concentration anomaly of heat capacity ...}

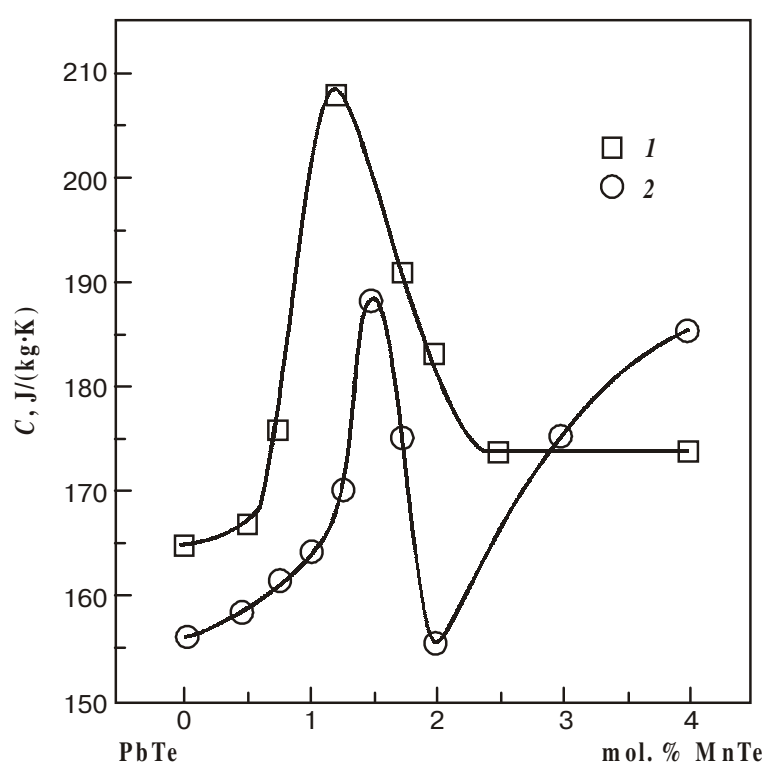

Fig. 2. Room-temperature heat capacity vs MnTe (1) and GeTe (2) content in the $\mathrm{Pb}_{1-\mathrm{x}} \mathrm{Mn}_{\mathrm{x}} \mathrm{Te}$ and $\mathrm{Pb}_{1-\mathrm{x}} \mathrm{Ge}_{\mathrm{x}} \mathrm{Te}$ solid solutions.

acteristics as correlation radius, heat capacity, etc. The difference consists in the fact that in accordance with the percolation theory the cluster boundaries are frozen, whereas in the II type phase transition theory they change due to thermal motion. The cluster density plays a role of the order parameter. One of the most typical features of a II type phase transition is sharp X-like maximum of $C$.

The most well-known among II-type phase transitions are temperature phase transitions. They include transitions from para- to ferro- or antiferromagnetic state at the Curie and Neel temperatures, respectively, below which magnetic ordering occurs; transitions from parato ferroelectric state at the Curie temperature, below which spontaneous electric polarization appears; transition of liquid helium into the superfluid state, the appearance of superconductivity in metals and semiconductors at certain temperatures. The II-type phase transition takes place in the critical point of vapor-liquid equilibrium or in the critical point of mixing of a two-component solution or under ordering of some alloys. All the above described phenomena possess common features. These are a change in symmetry in the transition point, an increase in the system susceptibility to external factors, the appearance of long-range ordering below the transition point, an $\lambda$-anomaly of the heat capacity. From the viewpoint of the fluctuation theory of II-type phase transitions, in the vicinity of the phase transition point, an anomalous growth of fluctuations that can not be considered as weak interactions, is observed. These strongly interacting fluctuations determine specific properties of the system, i.e. critical phenomena.

Significantly less studied are II-type concentration phase transitions. Among them are the Mott transition [11-13]; a transition to the ferromagnetic or ferroelectric state in a solid solution on reaching a critical concentra- tion of the impurity. In both cases, when a critical concentration is reached, there appears a new property in the solid solution, such as electrical conductivity, magnetization, polarizability, which plays a role of the order parameter. The mentioned concentration transitions are also considered in the framework of percolation theory [11-13]. For mathematical description of these phenomena an order parameter is introduced, which in lattice problems represents the fraction of lattice nodes $P(x)$ which belong to the infinite cluster. Near the percolation threshold, the $P(x)$ function has the following form: $P(x)=B_{1}\left(x-x_{c}\right)^{\beta}$ at $x>x_{c}$, and $P(x)=0$ at $x<x_{c}$ where $\beta$ is the critical index of the order parameter. Similar formulae describe the behavior of electrical conductivity, heat capacity, and other properties with corresponding critical indices.

At present, percolation theory is widely applied for description of electrical properties of disordered systems, such as amorphous semiconductors, crystal semiconductors with impurities and materials representing a mixture of two phases - dielectric and metallic. One of the major spheres of percolation theory applications is impurity doped semiconductors. In classical theoretical works on percolation, mainly static electrical conductivity and magnetization were studied.

In the systems studied in the present work, isovalent substitution takes place, i.e. the impurities are electrically inactive. However, impurity atoms are centers of local distortions of the crystal lattice, sources of internal stresses and strains, magnitudes of which are inversely proportional to the distance to the third power. Since noticeable displacements of atoms are created within the range of one or two interatom distances, one can treat the field of elastic strains as the short-range onr and consider the characteristic radius of deformational interactions $R_{0}$. At small impurity concentrations, when distances between impurity atoms are much greater than $R_{0}$, deformational fields created by individual atoms do not overlap and make additive contributions into such characteristics as microhardness and other mechanical properties, thermal resistance of the lattice, etc. As the concentration of impurity increases, elastic fields of neighboring atoms overlap, which leads to a partial compensation of elastic strains of opposite signs.

In the approximation of short-range interactions, assuming statistical distribution of impurity atoms and using the ideas of the percolation theory, one can estimate on the basis of $R_{0}$ values the concentration $x_{c}$ (percolation threshold), at which the formation of an uninterrupted chain of overlapping deformation fields penetrating the entire crystal (infinite cluster) occurs. One can also solve an opposite problem, i.e. knowing the value of the critical concentration $x_{c}$, calculate the radius of the impurity atom "action sphere" $R_{0}$ from the condition

$4 / 3 p N_{C}\left(2 R_{0}\right)^{3} \approx 2.7$,

where $N_{C}$ is the average number of sphere centers per volume unit. Assuming that $x_{c}$ corresponds to $\sim 0.012$ and 


\section{E.I. Rogacheva et al.: Concentration anomaly of heat capacity ...}

$\sim 0.015$ for $\mathrm{Pb}_{1-\mathrm{x}} \mathrm{Mn}_{\mathrm{x}} \mathrm{Te}$ and $\mathrm{Pb}_{1-\mathrm{x}} \mathrm{Ge}_{\mathrm{x}}$ Te solid solutions, respectively, we obtain $R_{0} \sim 1.4-1.2 a_{0}$, where $a_{0}$ is the unit cell parameter, which is in agreement with shortrange character of deformational interactions. In the case under consideration, percolation channels are formed through deformational fields of individual impurity atoms. The situation is similar to the Mott transition, when the formation of percolation channels through electron shells of individual atoms leads to the appearance of the electrical conductivity (metal-dielectric transition).

At critical concentrations the probability of the ordering of impurity atoms increases sharply. A simple calculation shows that the composition corresponding to $x \sim 0.01$ is optimal for the formation of a primitive cubic lattice of impurity atoms with the unit cell parameter $a=3 a_{0}$, while at $x \sim 0.016$ the formation of a superstructure representing a f.c.c. lattice of impurity atoms with the unit cell parameter $a=4 a_{0}$. Only after reaching the percolation threshold, ordering of impurity atoms becomes possible. In this case one can assume the formation of "impurity crystal".

One could assume that the peak of the heat capacity in the $\mathrm{Pb}_{1-\mathrm{x}} \mathrm{Ge}_{\mathrm{x}}$ Te system is connected with the manifestation of a concentration phase transition of a paraelectric-ferroelectric type, which takes place in this system under the introduction of a certain amount of noncentral ions of $\mathrm{Ge}$ into PbTe [21-25]. However, at Ge concentrations corresponding to $x<0.04$, such a transition occurs at temperatures much lower than room temperature [21-25]. Besides, the fact that the sharp growth in the heat capacity is also observed in the $\mathrm{Pb}_{1-\mathrm{x}} \mathrm{Mn}_{\mathrm{x}} \mathrm{Te}$ system, which does not possess ferroelectric properties, shows that the effect under consideration has a universal character and is not connected with properties of the introduced impurity.

As it follows from the obtained results, there is a sharp growth in the heat capacity in the interval of $x \approx 0.01-$ 0.015 , which is the evidence of a phase transition. Since in the range of solid solutions $x \approx 0-0.02$ the unit cell parameter decreases monotonically, the phase transition is of an isostructural type. It can be assumed that it has percolation nature and corresponds to the transition from "impurity gas" (region of diluted solid solutions) to "impurity condensate" (region of concentrated solid solutions). Since we observed anomalies of properties at small impurity concentrations in many solid solutions, one can suggest that this phenomenon has a universal character and inherent to any solid solution.

\section{Conclusions}

For the first time, anomalous growth in the heat capacity of the $\mathrm{Pb}_{1-\mathrm{x}} \mathrm{Mn}_{\mathrm{x}}$ Te and $\mathrm{Pb}_{1-\mathrm{x}} \mathrm{Ge}_{\mathrm{x}}$ Te solid solutions in the range of small impurity concentrations $(x \approx 0.012$ and 0.015 , respectively) was detected. The observed growth can be considered as a critical phenomenon. The assumption about the percolation nature of this effect is made. It is suggested that in any solid solution in the region of low concentrations of impurity the transition from "impurity gas" to "impurity condensate" takes place, i.e. chain of impurity atom interactions penetrating the whole crystal is formed. This transition can be considered as a II-type phase transition accompanied by anomalies of properties, in particular, an anomaly of the heat capacity.

The analysis of the obtained results as well as our previous data allows us to suppose that the existence of anomalies of properties in the range of small impurity concentration is a universal physical phenomenon in solid solutions of different types.

The appearance of concentration anomalies of properties (mechanical thermal, and electrophysical) under the transition from diluted to concentrated solid solutions, from slight to heavy doping, should be taken into consideration when developing new semiconductor materials including materials for infrared optoelectronics.

\section{Acknowledgements}

This work was supported by the Ministry of Education and Science of Ukraine

\section{References}

1. A.V. Lyubchenko, E.A. Salkov, F.F. Sizov, Physical principles of semiconductor infrared photoelectronics, Naukova Dumka, Kiev, 1984 (in Russian).

2. E.I. Rogacheva, I.M. Krivulkin, V.P. Popov, T.A. Lobkovskaya, Concentration dependences of properties in $\mathrm{Pb}_{1-\mathrm{x}} \mathrm{Mn}_{\mathrm{x}} \mathrm{Te}$ solid solutions // Phys. Status Solidi (A), 148(2), pp.K65-K67 (1995).

3. E.I. Rogacheva, N.A. Sinelnik, O.N. Nashchekina, Concentration anomalies of properties in $\mathrm{Pb}_{1-\mathrm{x}} \mathrm{Ge}_{\mathrm{x}} \mathrm{Te}$ solid solutions // Acta Phys. Polonica (A), 84(4), pp. 729-732 (1993).

4. E.I. Rogacheva, V.I. Pinegin, T.V. Tavrina, Percolation effects in $\mathrm{Pb}_{1-\mathrm{x}} \mathrm{Ge}_{\mathrm{x}} \mathrm{Te}$ solid solutions // Proc. SPIE, 3182, pp. 364-368 (1997).

5. E.I. Rogacheva, A.S. Sologubenko, I.M. Krivulkin, Microhardness of $\mathrm{Pb}_{1-\mathrm{x}} \mathrm{Mn}_{\mathrm{x}} \mathrm{Te}$ semimagnetic solid solutions // Inorganic materials, 34(6), pp. 545-549 (1998).

6. E.I. Rogacheva, T.V. Tavrina, and I.M. Krivulkin, Anomalous Composition Dependence of microhardness in $\mathrm{Pb}_{1-\mathrm{x}} \mathrm{Ge}_{\mathrm{x}} \mathrm{Te}$ semiconductors solid solutions // Inorganic materials, 35, pp.236-239 (1999).

7. E.I. Rogacheva, I.M. Krivulkin, Isotherms of charge carrier mobility in $\mathrm{Pb}_{1-\mathrm{x}} \mathrm{Mn}_{\mathrm{x}} \mathrm{Te}$ alloys // Proc. SPIE, 3950, pp. 18321838 (2000).

8. E.I. Rogacheva, I.M. Krivulkin, Изотермы теплопроводности в твердых растворах $\mathrm{PbTe}-\mathrm{MnTe} / /$ Fizica Tverdogo Tela, 43(6), pp. 1000-1003 (2001).

9. E.I. Rogacheva, Critical Phenomena in heavily-doped semiconducting compounds // Jpn. J. Appl. Phys., 32, Suppl. 32-3, pp. 775-777 (1993).

10. E.I. Rogacheva, Concentration-Dependent microhardness in semiconductor solid solutions // Izv. AN SSSR. Neorgan. Mater., 25(5), pp. 643-646 (1989.

11. A.L. Efros, Physics and geometry of disorder. Nauka Moscow, 1982.

12. B.I. Shklovskii and A.L. Efros, Electron Properties of Doped Semiconductors. Nauka Moskow, 1979.

13. D. Stauffer, Introduction to Percolation Theory. Taylor \& Fransis. London and Philadelphia, 1985. 


\section{E.I. Rogacheva et al.: Concentration anomaly of heat capacity ...}

14. A.Z. Patashinskiy, V.L. Pokrovskiy, The fluctuation theory of phase transitions. Nauka Moscow, 1982.

15. N.H. Abrikosov and L.E. Shelimova, Semiconducting materials based on $A^{2} B^{6}$ compounds, Nauka, Moscow, 1975 (in Russian).

16. Yu.1. Ravich, B.A. Efimova, and I.A. Smirnov, Semiconducting Lead Chalcogenides, Plenum Press, New York, London, 1970.

17. D.H. Parkinson, J.E. Quarrington, The molar heats of lead sulphide, selenide and telluride in the temperature range 20 K to $260 \mathrm{~K} / /$ Proc. Phys. Soc. A, 67, Part 7, N 415, pp. 569 579 (1954).

18. W. Cochran, R.A. Cowley, G . Dolling, M.M. Elcombe, The crystal dynamics of lead telluride // Proc. Royal Soc. A, 293, pp. 433-451 (1966).

19. A.J. Bevolo, H.R. Shanks, D.E. Eckels, Molar heat capacity of $\mathrm{GeTe}, \mathrm{SnTe}, \mathrm{PbTe}$ from 0.9 to $60 \mathrm{~K} / /$ Phys. Rev. B. V., 13(8), pp. 3523-3533 (1976)
20. S.I. Lykov, I.A. Chernik, Heat capacity of lead chalcogenides in the temperature range of 1-20 K // Fiz. Tverd. Tela, 24(10), pp. 3102-3105 (1982).

21. P.B. Littlewood, Phase transitions and optical properties of IV-VI compounds // Lect. Notes Phys., 152, pp.238-246 (1982).

22. T. Suski, Phase transitions and resistivity anomalies in $(\mathrm{Pb}$, Sn, Ge)Te compounds // Mater. Sci., 11(3), pp.3-53 (1985).

23. H. Bussmann-Holder, W. Bilz, Ferroelectric Phase Transition in $1 \mathrm{~V}-\mathrm{V} 1$ semiconductors // J. Phys. Soc. Jap., 49, Suppl.A., pp. 737-744 (1980)

24. D.K. Hohnke, H. Holloway, S. Kaiser, Phase relations and transpormations in the system $\mathrm{PbTe}-\mathrm{GeTe} / / \mathrm{J}$. of Physics and chemistry of solid, 33(12), pp. 2053-2057 (1972).

25. E.J. Fantner, H. Pascher, G. Bauer, R. Danzer, A. LopezOtero, Magnetooptical investigation of the Bandstructure of $\mathrm{Pb}_{1-\mathrm{x}} \mathrm{Ge}_{\mathrm{x}} \mathrm{Te}$ at the Structural Phase Transition $\left(\mathrm{O}_{\mathrm{h}}-\mathrm{C}_{3 \mathrm{~V}}\right) / / J$. Phus. Soc. Japan., Suppl. A, 49, pp. 741-744 (1980). 